\title{
Visual Functional Defects in Patients with type 2 Diabetes Mellitus: A Questionnaire Based Cross- Sectional Study
}

Yimeng Ge ( $\nabla$ gym_susan@pku.edu.cn )

Peking University Third Hospital https://orcid.org/0000-0001-8939-8681

\section{Sanbao Chai}

Peking University International Hospital

\section{Yu Wan}

Peking University Third Hospital

Huaqin Xia

Peking University Third Hospital

\section{Ruilan Dong}

Peking University Third Hospital

\section{Qingyi Hou}

Peking University Third Hospital

\section{Xiaotong Ren}

Peking University Third Hospital

\section{Hao Yuan}

Peking University Third Hospital

\section{Jiarui Yang}

Peking University Third Hospital

\section{Xuemin Li}

Peking University Third Hospital

\section{Research Article}

Keywords: Type 2 diabetes, visual functions, retinopathy, quality of life

Posted Date: September 30th, 2021

DOI: https://doi.org/10.21203/rs.3.rs-925224/v1

License: (c) (1) This work is licensed under a Creative Commons Attribution 4.0 International License.

Read Full License 
1 Visual functional defects in patients with type 2 diabetes mellitus: a questionnaire based

2 and approved the final draft of the manuscript submitted.

\section{Funding}




\section{Compliance of ethical standards}

22

23

Informed consent was obtained from all individual participants included in the study.

\section{Purpose}

To determine the impact of type 2 diabetes mellitus (T2DM) on visual functions, identify different modifiers as risk or protective factors, and find out how these factors affect patients' visual symptoms and vision-related quality of life as a whole.

\section{Methods}


Demographic features and severity of disease were documented, while visual functions were evaluated using National Eye Institute Visual Functioning questionnaire-25 (NEI VFQ-25). Independent t-test, analysis of variance, linear and non-linear regression models were used to assess all data.

\section{Results}

Scores other than color vision among T2DM patients were significantly lower compared with non-T2DM participants. There was significant difference after stratification of age and education, but no significant difference between different genders was observed. Parameters including duration of T2DM, fasting plasma glucose (FPG) and glycosylated hemoglobin A1c (HbA1c) negatively impacted on the scores, with 20 years' of diabetic duration, $10 \mathrm{mmol} / \mathrm{L}$ of $\mathrm{FPG}, 7.5 \%$ of $\mathrm{HbA} 1 \mathrm{c}$ being potential cut-off points. Poorer best corrected visual acuity (BCVA) and diagnosis of diabetic retinopathy were risk factors, while they simultaneously produced mediation effect, contributing 5\%-78\% of effect in the deterioration of visual functions caused by longer diabetic duration and higher blood glucose.

\section{Conclusion}

Significant visual impairments and faster deterioration in visual functions were seen in T2DM patients, with older age, lower educational level, longer diabetic duration, poorer blood glucose administration, limited BCVA, and the presence of diabetic retinopathy identified as risk factors. Average BCVA and diabetic retinopathy also yielded mediation effect as diabetic duration lengthened and blood glucose elevated. 
57 Type 2 diabetes, visual functions, retinopathy, quality of life

\section{Introduction}

Type 2 Diabetes Mellitus (T2DM) is one of the most common yet severe metabolic diseases affecting millions of people worldwide ${ }^{[1]}$. With global, regional and country-level estimates, it is predicted that the prevalence, mortality rate and health expenditure are all likely to go through a dramatic increase in both developed and developing countries ${ }^{[2]}$. Additionally, T2DM is associated with multiple ophthalmologic complications, specifically diabetic retinopathy ${ }^{[3]}$ and diabetic macular edema ${ }^{[4]}$, which are thought to severely impact on patients' vision and thus led to decreased vision-related quality of life and other adverse clinical outcomes. Other minor visual problems related to alterations in refractive error, contrast sensitivity, straylight and presbyopia also contributed to visual impairments and disturbance in T2DM patients. As estimated by the WHO Multinational Study of Vascular Disease in Diabetes (WMSVDD) in a 8.4-year follow-up covering 10 centers across the globe, the cumulative incidence of mild, moderate and severe visual impairments in T2DM patients were 9.43\%, 3.21\% and $2.25 \%$ respectively ${ }^{[5]}$. Therefore, the evaluation of visual function should serve as an importance component to assess the well-being of T2DM patients and be treated as valuable trackers or predictors that promote the overall administration of T2DM.

In prior studies, multiple rating scales featuring visual functional has already been administered to 
T2DM patients with a few modifiers (e.g. old age, male gender) identified as risk factors. However, a comprehensive analysis of different influence factors of visual functions and how these factors collaboratively contribute to the progression of T2DM is still lacking. Our study aims to characterize the pattern of visual impairment among T2DM patients in comparison with individuals free of T2DM, identify certain demographic and disease-related features as protective or risk factors and analyze how they interfere with each other and promote the overall development and prognosis of T2DM.

\section{Materials and methods}

\subsection{Study Design}

\section{With approval from the Ethics Committee of Peking University Third Hospital, a questionnaire was} sent out to patients and volunteers via the internet. The questionnaire was divided into three parts, with the first part collecting basic information (age, gender, educational level), the second part featuring severity of disease (diabetic duration, fasting glucose, glycosylated hemoglobin A1c, best corrected visual acuity and the presence of diabetic retinopathy), and the last part determined visual performance of patients using NEI VFQ-25, which was considered as a valid tool demonstrating multiple levels of functional defects and inconvenience in conducting daily tasks among patients with chronic ophthalmologic diseases ${ }^{[3,6]}$.

\subsection{Study participants}

In this study, questionnaires were distributed through the Internet between June 12, 2020 and August 30, 
2020. A total of 1244 individuals were screened, 400 were T2DM, and 844 were non-T2DM, which yielded a representative sample for the respective communities. Further stratification was done based on patients' age and duration of T2DM. 9 groups were formed in all participants with each group possessed an age range of 10 years, while duration of T2DM were classified as 0-5 years, 5-10 years, 10-20 years, $20-30$ years, and $>30$ years.

\subsection{Information Collection}

The data of basic information, diabetic-related information, vision quality and vision-related quality of life were collected. The duration of T2DM were considered as the time between initial diagnosis and the time when NEI VFQ-25 was administered to the patient, while fasting plasma glucose (FPG) and glycosylated hemoglobin Alc (HbAlc) were collected based on patients' self-reported data of recent clinical examinations. Best corrected visual acuity (BCVA) of both eyes were specified as the result from patients' last clinical visit. Decimal visual acuity, which is currently widely applied in clinical practice in China, was collected, while the data was transformed to LogMAR for analysis. Meanwhile, the presence of diabetic retinopathy was confirmed as patients having previous diagnosis in proper healthcare settings.

The scores of NEI VFQ-25 of all participants were calculated according to the following procedures.

Patients were required to rate their performance in vision-related tasks with a 5 or 6 point scale ranging from "having no difficulties at all" to "not being able to do this because of eyesight". A score of 0-100 were then given to each answer as 100 being the optimum and 0 being the worst. An answer of "stop 
111 doing this for other reasons or not interested in doing this" was considered to be missing data. The

112 scores of 6 sub-scales were calculated as the average of scores for each question under this sub-scale,

113 while a total score was obtained through averaging of all sub-scale scores ${ }^{[7]}$.

\subsection{Statistical Analysis}

115 Statistical analysis was performed by using SPSS version 26.0 and R 4.0.4. The continuous variable

116 and categorical variables were presented as mean \pm standard deviation (SD) and frequency (\%),

117 respectively. Independent t-test and analysis of variance were used to assess the data between T2DM

118 and control group while a $P$-value $<0.05$ was considered to be significant. Moreover, we applied a

119 non-linear regression model to describe the changes of vision-related in all sub-scales among two

120 groups with age. We also reported the changing of NEI VFQ-25 scores induced by aging, lengthening

121 of diabetic duration, elevation of FPG and HbA1c as well as deterioration of eyesight with restricted

122 cubic spline (smooth curve). Further mediation effect of average vision and baseline diabetic

123 retinopathy were assessed using mediation effect analysis.

\section{Results}

\subsection{Basic data}


129 without T2DM. 23.7\% patients with T2DM were already diagnosed with retinopathy, whereas the

130 average BCVA was $0.22 \pm 0.31$ and $0.19 \pm 0.28$ for the left and the right eye respectively.

131 TABLE 1 Baseline characteristics of T2DM patients and Non-T2DM participants

\begin{tabular}{llll}
\hline & T2DM & Non-T2DM & p-value \\
Characteristics & $(\mathbf{n = 4 0 0 )}$ & $\mathbf{( n = 8 4 4 )}$ & \\
\hline Age,years & $59.01 \pm 11.38$ & $41.64 \pm 12.76$ & $<0.001 * * *$ \\
Gender & & & \\
Male & 188 & 318 & $<0.001 * * *$ \\
Female & 212 & 526
\end{tabular}

Educational level

Illiteracy 10

Primary School $\quad 30 \quad 6$

Secondary School $\quad 89 \quad 40$

$\begin{array}{lll}\text { High School } & 102\end{array}$

$\begin{array}{lll}\text { Undergraduate } & 157 & 576\end{array}$

$\begin{array}{lll}\text { Graduate or above } & 12 & 87\end{array}$

Diabetic duration,years $\quad 11.02 \pm 8.02$

FPG, $\mathrm{mmol} / \mathrm{L} \quad 8.16 \pm 4.00$

$\mathrm{HbA1c, \%} \quad 8.23 \pm 5.85$

BCVA

\begin{tabular}{|c|c|c|c|}
\hline Left & $0.22 \pm 0.31$ & $0.15 \pm 0.18$ & $<0.001 * * *$ \\
\hline Right & $0.19 \pm 0.28$ & $0.09 \pm 0.11$ & $<0.001 * * *$ \\
\hline
\end{tabular}


Diabetic Retinopathy

Yes

No
71

229
132

134

135

136

$\mathrm{n}=$ number, $\mathrm{T} 2 \mathrm{DM}=$ Type 2 diabete mellitus, $\mathrm{FPG}=$ fasting plasma glucose, $\mathrm{BCVA}=$ best corrected visual acuity, HbA1c= glycosylated hemoglobin A1c

$p<0.001$ was marked with ***.

\subsection{Functional defects in T2DM patients and Non-T2DM participants}

Both groups completed the questionnaire and visual functional defects in all participants were relatively slight since mean scores for most sub-scales exceeded 90, demonstrating satisfactory performance in all participants. Notably, the scores for "General Vision", which was based on the personal assessment of vision-related quality of life, were comparably lower in both groups, indicating the need of alleviating visual symptoms or bettering visual performance even in participants not suffering from T2DM.

As shown in Table 2, the total and mean scores for 5 NEI VFQ-25 sub-scales scores were lower in T2DM group $(p<0.001$, independent t-test). However, there was no significant difference between the two groups in the scores of color vision. Furthermore, after stratification by age, gender and educational level, we found that the performance of elderly patients with T2DM were worse than those non-T2DM participants of the same age, in terms of distance activities ( $p=0.033)$, social functioning $(p=0.006)$, peripheral vision $(p=0.025)$ and total score $(p=0.013)$ (Supplementary Table 1$)$. Similar 


\section{TABLE 2 Visual functional defects among T2DM patients and non-T2DM participants}

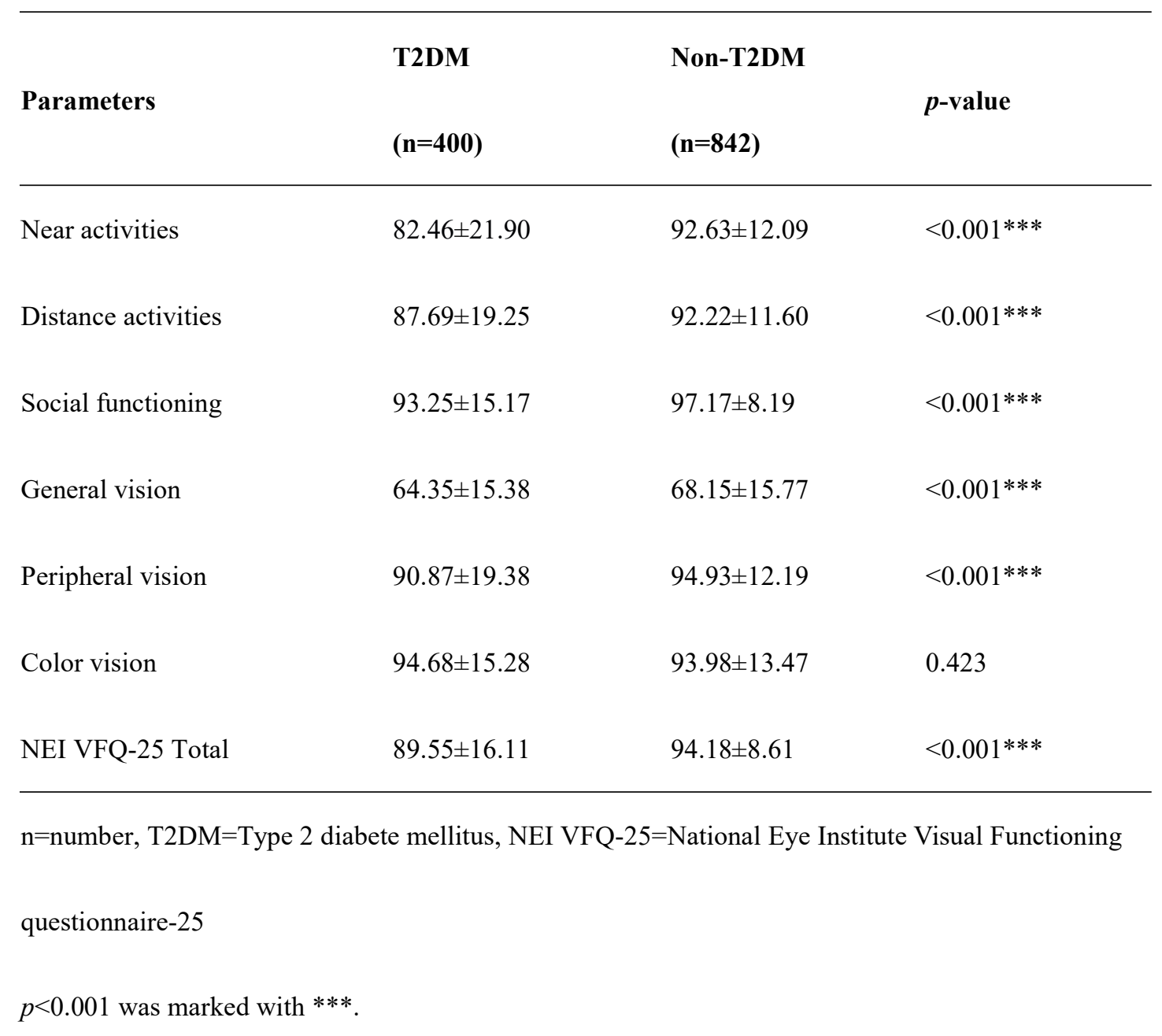

157 $\mathrm{n}=$ number, T2DM=Type 2 diabete mellitus, NEI VFQ-25=National Eye Institute Visual Functioning questionnaire-25

stratification was done with regard to gender and educational level. These results indicated that all sub-scales and total score in T2DM patients were lower than non-T2DM participants except for color vision after stratification by gender (Supplementary Table 2), while significant results were only seen in participants sharing an educational level of secondary school, high school and undergraduate (Supplementary Table 3$)$. The scores of near activities $(p=0.007)$ and distance activities $(p=0.021)$ of T2DM with secondary school education background were lower than non-T2DM counterparts.

Undergraduate T2DM patients, however, reported greater functional defects in near activities and social functioning with statistical significance $(p<0.001, p=0.012)$ compared with non-T2DM participants. 
160

161

162

163

164

165

166

167

a

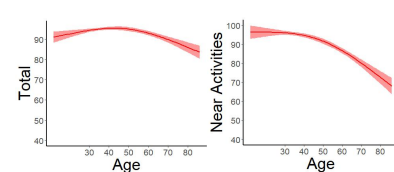

b

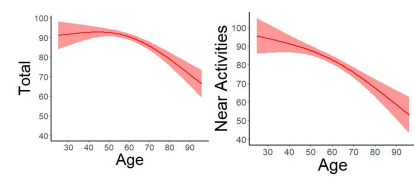

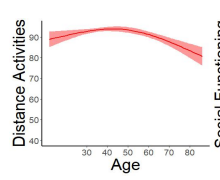
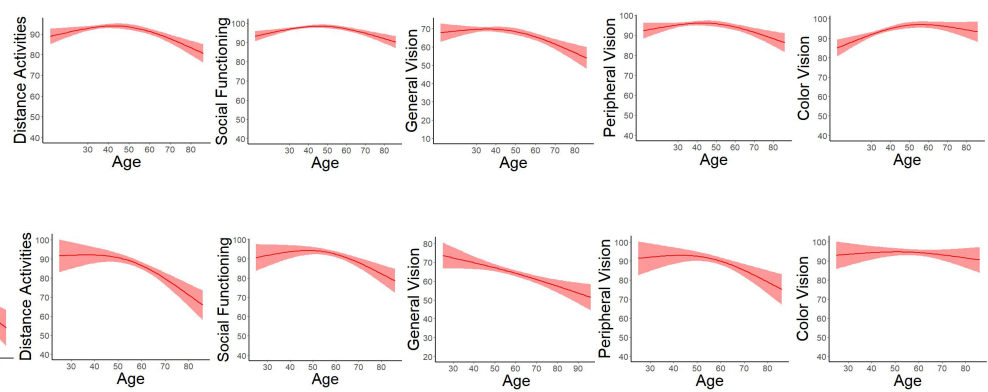

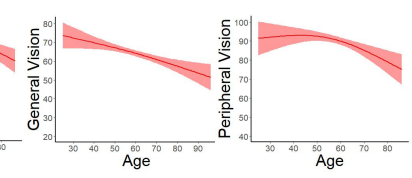

FIGURE 1 Correlation between visual functions and aging in T2DM patients (a) and non-T2DM

participants (b). We used restricted cubic spline to characterize the non-linear correlation between 
178 When demographic variables of T2DM patients, including age, gender and educational level were examined independently, we found that all these modifiers contributed to the overall effect of the hyperglycemic metabolic disorder (Table 3). In all sub-scales other than color vision, older age and lower educational level contributed to lower scores of NEI VFQ-25, therefore indicating higher risks of visual functional loss and heavier dependence on caregivers in vision-related tasks. Female patients exhibited poorer visual performance, however, we found that gender had no effect on the decline of visual function after matching female and male patients with age. Among all demographic features, the age of patients had the greatest impact on visual impairment, as the $F$-value for age, gender and education level were $6.592,3.026$, and 1.068 , respectively. In the color vision sub-scale, on the contrary, none of all the demographic features were shown to leave either positive of negative effect.

TABLE 3 Visual functions in T2DM patients stratified by demographic features

\begin{tabular}{|c|c|c|c|c|c|c|c|}
\hline \multirow{3}{*}{ Subscales } & Near & Distance & Social & General & Peripheral & Color & \multirow{3}{*}{ Total } \\
\hline & & & & & & & \\
\hline & Activities & Activities & Functioning & Vision & Vision & Vision & \\
\hline \multicolumn{8}{|l|}{ Age } \\
\hline $20-29$ & 95.83 & 91.67 & 93.75 & 70.00 & 87.50 & $100.00 \pm$ & $93.75 \pm 8$ \\
\hline$(n=2)$ & \pm 5.89 & \pm 11.79 & \pm 8.84 & \pm 14.14 & \pm 17.68 & 0.00 & .84 \\
\hline $30-39$ & 92.95 & 93.27 & 95.67 & 72.31 & 92.31 & 96.00 & 93.93 \\
\hline$(n=26)$ & \pm 15.58 & \pm 12.25 & \pm 11.15 & \pm 15.05 & \pm 13.73 & \pm 11.81 & \pm 11.44 \\
\hline
\end{tabular}




\begin{tabular}{|c|c|c|c|c|c|c|c|}
\hline $40-49$ & 90.85 & 93.44 & 93.88 & 68.09 & 95.21 & 93.18 & 93.38 \\
\hline$(n=47)$ & \pm 16.14 & \pm 15.14 & \pm 11.15 & \pm 16.50 & \pm 14.41 & \pm 20.43 & \pm 15.04 \\
\hline $50-59$ & 84.46 & 90.09 & 95.16 & 64.86 & 92.79 & 95.27 & 91.55 \\
\hline$(\mathrm{n}=111)$ & \pm 18.00 & \pm 15.06 & \pm 13.18 & \pm 14.82 & \pm 16.30 & \pm 12.84 & \pm 12.83 \\
\hline $60-69$ & 81.97 & 88.92 & 94.84 & 63.89 & 92.48 & 95.60 & 90.74 \\
\hline$(n=144)$ & \pm 22.39 & \pm 17.94 & \pm 12.35 & \pm 15.20 & \pm 19.01 & \pm 14.37 & \pm 14.51 \\
\hline $70-79$ & 71.53 & 75.68 & 87.09 & 59.34 & 81.25 & 91.67 & 81.35 \\
\hline$(n=61)$ & \pm 26.05 & \pm 24.62 & \pm 18.95 & \pm 14.59 & \pm 24.63 & \pm 18.79 & \pm 18.69 \\
\hline $80-89$ & 67.86 & 70.23 & 71.43 & 57.14 & 78.57 & 95.00 & 73.78 \\
\hline$(n=7)$ & \pm 28.23 & \pm 43.53 & \pm 31.22 & \pm 13.80 & \pm 39.33 & \pm 11.18 & \pm 33.86 \\
\hline \multicolumn{8}{|l|}{$90-99$} \\
\hline & 12.5 & - & - & 40 & - & - & 12.5 \\
\hline & & & & & & & $<0.001^{*}$ \\
\hline \multirow[t]{2}{*}{$p$-value } & $<0.001 * * *$ & $<0.001 * * *$ & $<0.001 * * *$ & $0.004 * *$ & $0.001 * *$ & 0.699 & \\
\hline & & & & & & & $* *$ \\
\hline \multicolumn{8}{|l|}{ Gender } \\
\hline Male & 86.25 & 91.01 & 94.65 & 67.02 & 93.05 & 95.30 & 91.98 \\
\hline$(\mathrm{n}=188)$ & \pm 18.11 & \pm 16.49 & \pm 12.49 & \pm 15.54 & \pm 16.75 & \pm 14.63 & \pm 13.46 \\
\hline Female & 79.11 & 84.74 & 92.00 & 61.98 & 88.93 & 94.14 & 87.40 \\
\hline$(\mathrm{n}=212)$ & \pm 24.34 & \pm 21.01 & \pm 17.14 & \pm 14.89 & \pm 21.31 & \pm 15.84 & \pm 17.89 \\
\hline$p$-value & $0.001 * *$ & $0.001 * *$ & 0.077 & $0.001 * *$ & $0.032 *$ & 0.451 & $0.004 * *$ \\
\hline \multicolumn{8}{|c|}{ Educational } \\
\hline
\end{tabular}




\begin{tabular}{|c|c|c|c|c|c|c|c|}
\hline Illiteracy & 54.17 & 66.67 & 83.75 & 50.00 & 62.50 & 86.11 & 70.13 \\
\hline$(\mathrm{n}=10)$ & \pm 32.45 & \pm 26.06 & \pm 16.72 & \pm 23.57 & \pm 29.46 & \pm 18.16 & \pm 19.57 \\
\hline \multicolumn{8}{|l|}{ Primary } \\
\hline & 70.09 & 78.89 & 87.5 & 60.67 & 84.17 & 90.18 & 82.27 \\
\hline \multicolumn{8}{|l|}{ School } \\
\hline & \pm 28.76 & \pm 24.34 & \pm 24.34 & \pm 16.17 & \pm 24.99 & \pm 24.85 & \pm 22.47 \\
\hline \multicolumn{8}{|l|}{$(\mathrm{n}=30)$} \\
\hline \multicolumn{8}{|l|}{ Secondary } \\
\hline & 79.07 & 86.84 & 93.47 & 62.25 & 92.82 & 94.54 & 88.58 \\
\hline \multicolumn{8}{|l|}{ School } \\
\hline & \pm 23.47 & \pm 18.82 & \pm 12.57 & \pm 16.08 & \pm 16.13 & \pm 13.44 & \pm 16.69 \\
\hline \multicolumn{8}{|l|}{$(n=87)$} \\
\hline \multicolumn{8}{|l|}{ High } \\
\hline & 82.39 & 88.19 & 92.89 & 64.12 & 90.67 & 95.05 & 89.85 \\
\hline \multicolumn{8}{|l|}{ School } \\
\hline & \pm 21.48 & \pm 19.66 & \pm 15.98 & \pm 14.44 & \pm 20.19 & \pm 14.15 & \pm 15.91 \\
\hline \multicolumn{8}{|l|}{$(\mathrm{n}=106)$} \\
\hline \multicolumn{8}{|l|}{ Undergra } \\
\hline & 87.85 & 90.39 & 94.63 & 66.62 & 92.63 & 95.75 & 92.13 \\
\hline \multicolumn{8}{|l|}{ duate } \\
\hline & \pm 16.65 & \pm 17.00 & \pm 13.77 & \pm 14.03 & \pm 17.56 & \pm 14.84 & \pm 13.38 \\
\hline \multicolumn{8}{|l|}{$(\mathrm{n}=152)$} \\
\hline \multicolumn{8}{|l|}{ Graduate } \\
\hline & 90.28 & 93.75 & 98.96 & 73.33 & 95.83 & 95.83 & 94.93 \\
\hline \multicolumn{8}{|l|}{ or above } \\
\hline & \pm 12.73 & \pm 8.79 & \pm 3.61 & \pm 15.57 & \pm 9.73 & \pm 9.73 & \pm 7.00 \\
\hline \multicolumn{8}{|l|}{$(n=12)$} \\
\hline & & & & & & & $<0.001^{*}$ \\
\hline \multirow[t]{2}{*}{$p$-value } & $<0.001 * * *$ & $<0.001 * * *$ & $0.045^{*}$ & $0.001 * *$ & $<0.001 * * *$ & 0.293 & \\
\hline & & & & & & & $* *$ \\
\hline $\mathrm{n}=$ number & & & & & & & \\
\hline
\end{tabular}


192 Moreover, we evaluated the duration of T2DM, FPG and HbA1c level, which were key factors

193 determining the degree of T2DM to explore their impact on the vision function related to quality of life.

194 As shown in Figure 2A, the duration of T2DM negatively correlated with scores of each sub-scale and total score of NEI VFQ-25 except for color vision. The scores of patients with a course of more than 20 years decreased significantly, suggesting that 20 year is a significant cut-off point. With the extension of the course of diabetes, the scores of near activities decreased gradually and might serve as a more sensitive parameter in evaluating visual loss. wasn't observed until patients' FPG reached 10mmol/l, whereas scores for general vision didn't differ much when patients' FPG exceeded $15 \mathrm{mmol} / 1$, as relative lower FPG level and better control of T2DM's hyperglycemic effect might elevate patients' expectation in vision-related quality of life and lower their general satisfaction regarding visual functions. As an indicator of blood glucose control in recent 3 months, HbA1c will also affect visual function. 
and HbA1c level had influence on scores of NEI VFQ-25. However, duration of T2DM produced the with FPG ( $F=1.344$, multivariate analysis $)$ and $\mathrm{HbA1c}(F=1.069)$.

214 Moreover, the correlation between the average BCVA of both eyes, the presence of retinopathy and

215 visual functional loss were examined using both non-linear regression model and independent $\mathrm{t}$-test. As

216 shown in Figure 2D, scores for each sub-scale and total score were found to decrease as BCVA worsened, with potential cut-off point identified at an average BCVA of 0.4. Color vision, however,

218 weren't shown to display either positive or negative correlation with average BCVA of both eyes. The

219 presence of diabetic retinopathy (DR), which was the main symbol for micro-vessel degeneration

a
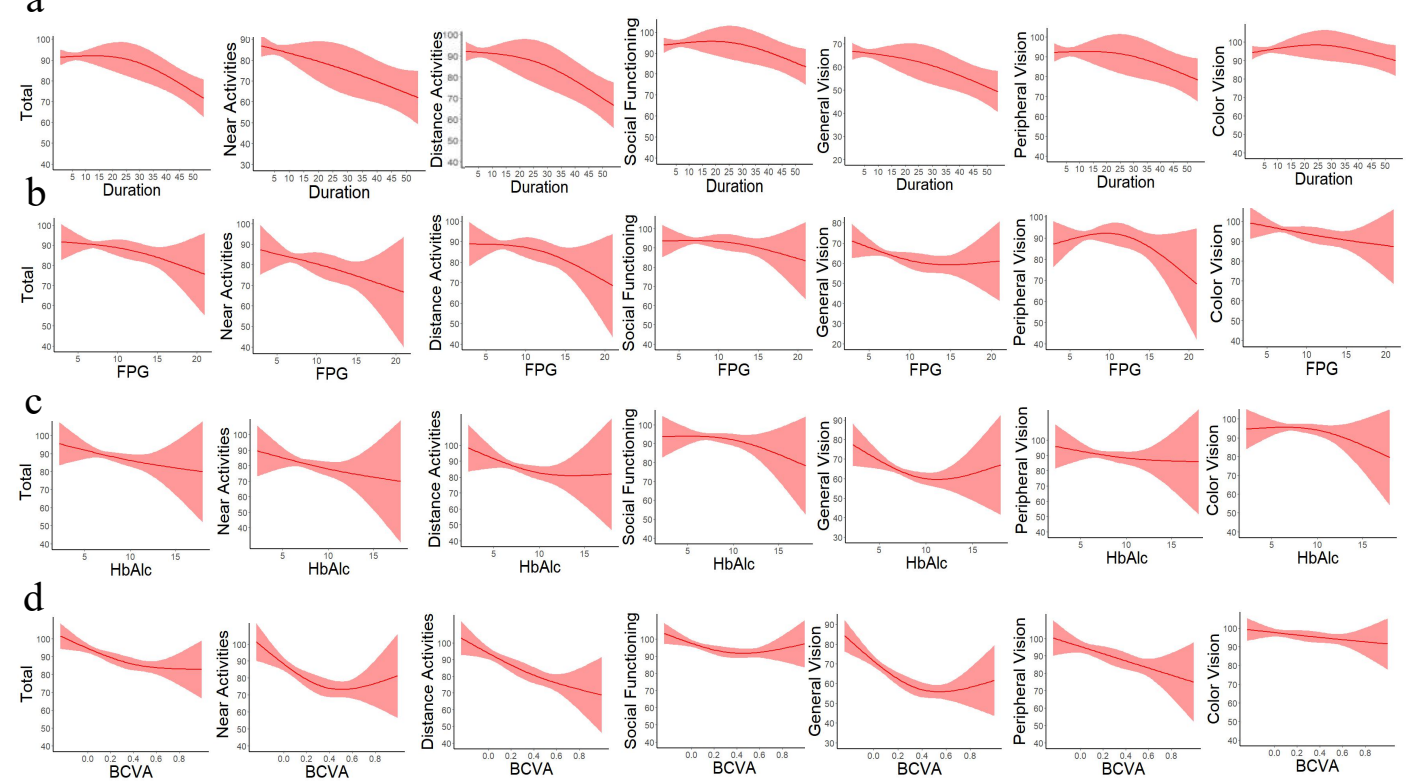
and average vision (d) Smooth curves along with 95\%CI were demonstrated above indicating the progression of functional loss in each vision-related sub-scales as duration of T2DM lengthened, FPG and $\mathrm{HbAlc}$ elevated and average vision deteriorated.

In general, older age, lower educational, long duration of T2DM, elevated FPG and HbA1c level were identified risk factors involved in visual impairment, whereas age and duration of T2DM were the two most important factors. Poorer BCVA and the presence of diabetic retinopathy, on the other hand, were not only independent risk factors, but also essential mediators in the long-term effect of longer duration of T2DM, higher FPG and HbA1c level.

\subsubsection{Mediation effect analysis of different modifiers}

Through analysis of relationship between diabetic duration, BCVA and scores for sub-scales as well as total scores of NEI VFQ-25, we found that longer duration negatively impacted on T2DM patients' multiple visual functions through deterioration of average BCVA, and mean proportion of the mediation effect in different sub-scales ranged from $19.20 \%$ to $42.70 \%$. Similar mediation effect was found in FPG, HbA1c and NEI VFQ-25. The proportion of mediation effect was shown in Table 4, which pointed out that the mediation effect of average BCVA was more statistically significant than the presence of diabetic retinopathy Furthermore, color vision and social functioning sub-scale were the least likely to be influenced by the mediation effect of the two ophthalmologic parameters. General vision on the contrary, was demonstrated to have BCVA and the presence of retinopathy share the 
242 largest proportion of contribution, as subjective thoughts towards one's general vision might be the

243 most sensitive to subtle changes in eyesight and was the earliest sub-scale to be influenced by the

244 diagnosis of diabetic retinopathy.

245 TABLE 4 Mediation effect of BCVA and diabetic retinopathy

\begin{tabular}{|c|c|c|c|c|c|c|c|}
\hline Mediation & Near & Distance & Social & General & Peripheral & Color & Total \\
\hline $\begin{array}{l}\text { Effect } \\
(\%)\end{array}$ & Activities & Activities & Functioning & Vision & Vision & Vision & Score \\
\hline \multicolumn{8}{|l|}{ Diabetic } \\
\hline duration-BC & $19.93 * * *$ & $28.06 * * *$ & $19.20 *$ & $36.40 * *$ & $42.70 * *$ & 20.89 & $29.22 * *$ \\
\hline \multicolumn{8}{|l|}{ VA } \\
\hline FPG-BCVA & $47.30 * * *$ & $46.60 * * *$ & $51.70 * * *$ & $58.20 * * *$ & $54.50 * * *$ & $25.40 * *$ & $62.00^{*}$ \\
\hline \multicolumn{8}{|l|}{ HbAlc-BCV } \\
\hline \multicolumn{8}{|l|}{ A } \\
\hline \multicolumn{8}{|l|}{ Diabetic } \\
\hline duration-Ret & 77.6 & 49.01 & 63.42 & 33.00 & 61.57 & 52.91 & 73.77 \\
\hline \multicolumn{8}{|l|}{ inopathy } \\
\hline \multicolumn{8}{|l|}{ FPG-Retino } \\
\hline \multicolumn{8}{|l|}{ pathy } \\
\hline \multicolumn{8}{|l|}{ HbA1c-Reti } \\
\hline nopathy & 19.20 & 11.90 & 13.69 & 60.20 & 5.97 & 5.79 & 15.50 \\
\hline
\end{tabular}

$246 \mathrm{FPG}=$ Fasting plasma glucose, $\mathrm{HbA} 1 \mathrm{c}=$ glycosylated hemoglobin $\mathrm{A} 1 \mathrm{c}, \mathrm{BCVA}=$ best corrected visual 
acuity

$p<0.05$ was marked with* $p<0.01$ was marked with** $p<0.001$ was marked with ${ }^{* * *}$

\section{Discussion}

250 Our study presented a cross sectional survey that examined visual functional loss in T2DM patients, identified risk factors as well as protective factors that modified patients' performance. We found that visual functional loss was more common and significant in T2DM patients compared with non-T2DM participants, with the scope of visual impairment in T2DM patients shown to be larger and the progression comparably faster. Old age, low educational, long duration of T2DM, elevated blood glucose level (FPG and $\mathrm{HbA1c}$ ), poor BCVA and the presence of diabetic retinopathy negatively age , 20 years of diabetic duration, $10 \mathrm{mmol} / \mathrm{L}$ of FPG, and $7.5 \%$ of $\mathrm{HbAlc}$ might be the cut-off point of vision decline. Average vision and the presence of retinopathy, on the other hand, were either independent modifiers and essential mediators that mediated the effect of diabetic duration, FPG and HbA1c level. non-T2DM participants, while the visual functions of T2DM patients decreased more significantly as age increased. As reported in prior studies, older patients were more susceptible to stressors contributing to diabetic vascular changes, including oxidative stress, metabolic products and other toxic transmitters ${ }^{[3,8,9]}$. Patients of older age also suffer from more complicated comorbidities and geriatric 
rapid impairments of functional loss in vision-related activities ${ }^{[10]}$.

Applying a non-linear regression model, we identified that both T2DM patients and non-T2DM participants exhibited a single-peak shaped pattern of functional loss as age grew. Younger patients, however, weren't shown to perform better than patients in their 40s or 50s. For T2DM patients, on the one hand, this single peak-shaped correlation might be explained with the fact that those who developed T2DM at younger age probably suffer from more severe eye disease and yet were more sensitive to functional loss in vision-related activities. As reported by Song et $\mathrm{al}^{[11]}$, the prevalence and degree of visual loss in T2DM increased substantially after patients reached their middle age, while studies featuring younger population indicated that diabetic retinopathy in prepubescent children, teenagers and young adults might be of higher severity and faster progression since insulin requirements in adolescence were relatively high, and teenage hormonal axis were immature and less sensitive to drugs ${ }^{[12]}$. On the other hand, younger non-T2DM patients might generally go through more frequent yet complicated visual tasks and required higher vision related quality of life compared with senior participants, which helps to explain why young participants scored lower than middle-aged participants. In the general vision sub-scale however, there was no significant differences in scores between two groups, which could possibly be explained by the fact that non-T2DM participants were more involved in vision-related tasks and thus set a higher standard for maintaining satisfactory visual performance. We concluded that both adolescent and senior T2DM patients should be paid with special attention as their physical condition featured more complicated comorbidities, higher hormone requirements and lower compliance, which therefore called for strict glycemic control and systematic disease administration. 
Paralleled to prior studies, both old age and low educational level were thought to be risk factors predicting poorer visual functions and vision-related quality of life ${ }^{[2,13,14]}$. Effect of other demographic features such as gender varied in different cohorts. In 2013, a study in Japan found that female participants had a higher prevalence of diabetic retinopathy at baseline and were more likely to develop severe retinopathy with the development of diabetes ${ }^{[15]}$. However, more studies suggested that the male sex was an independent risk factor for the high prevalence and progression, emphasizing the protective effect of estrogen and identifying testosterone and androgen as detrimental to diabetic micro-vascular complications $^{[16,17]}$.

For parameters determining severity of T2DM, duration of disease, elevated FPG and HbA1c level were generally found to negatively impacted on visual performances. Nonetheless, different edge points were confirmed in different studies. Olivarius et al used a FPG 10 and $17 \mathrm{mmol} / \mathrm{L}$ along with a $\mathrm{HbA} 1 \mathrm{c} 9 \%$ and $11 \%$ respectively, whereas Foo et al ascertained a transformation zone of HbAlc $7 \%-8 \%[14,18]$. The variation in the selection of the cut-off point for HbAlc could be explained by the different study procedures in two cohorts. Olivarius et al on the one hand, used average vision as the major parameter assessing visual loss, while on the other hand, moderate diabetic retinopathy was seen as the observational outcome in the latter report ${ }^{[14]}$. Moderate diabetic retinopathy, diagnosed by proliferation of micro-vessels through fundus photography, led to a lower cut-off point of HbAlc, with subjective visual loss being less sensitive and were unable to give an early warning of visual loss until HbAlc reached $9 \%{ }^{[14,18]}$. Therefore, we suggested that fundus degeneration symbolized by vascular changes, which was sensitive to hyperglycemic conditions, might accounted for the low scores in relative sub-scales as diabetic duration lengthened and blood glucose level elevated. Moreover, 20 
years' of diabetic duration were regarded as probable cut-off point in a Tunisian cohort conducted in $2014^{[19]}$, while Liu et al reported 15 years to be a meaningful stage symbolizing severe ocular degeneration ${ }^{[20]}$. Such differences were possibly due to the different definitions of visual loss, as outcome indicators were subjective visual symptoms in some studies while others used rating scales or ocular examinations as diagnostic tools. In our study, both subjective evaluation of visual loss and objective tools determining BCVA were applied, with more detailed definition of different types of visual functional defect and degree of decreased vision-related quality of life using the NEI VFQ-25 rating scale. Such combination of diagnostic tools defined relatively sensitive cut-off point of HbAlc and FPG level, while the cut-off point of T2DM duration were not as sensitive as expected. This emphasized the need for appropriate yet comprehensive combination of different diagnostic tools, and also early intervention of T2DM as no golden standard of cut-off points were so far established.

Additionally, the insignificant effect of early elevation of FPG and HbAlc were possibly due to the fluctuation of long term glycemic control, as related studies stated that the oscillation of blood glucose boasted higher predictive value rather than parameters determining average blood glucose level ${ }^{[21,22]}$.

Ocular parameters, namely average BCVA and baseline retinopathy in our study, were considered as essential modifiers of visual functions. Poorer vision and the existence of ocular vascular degeneration limited the scale and acuity of optesthesia in T2DM patients thus disturbed their vision-related quality of life in several ways including elevating intraocular pressure and damaging nerve fiber layers ${ }^{[19,23]}$. However, some reports revealed the protective effect of myopia on the development of diabetic retinopathy, which may indicate that patients suffered from myopia were less likely to develop diabetic retinopathy, but such protective effect disappeared once diabetic retinopathy ensued. 
The mediation effect of average BCVA and baseline diabetic retinopathy, accounted for different proportions of functional loss in various NEI VFQ-25 sub-scales. General vision was the most sensitive sub-scale influenced by poor eyesight, as participants mainly judge their autonomous performance in vision-related daily tasks based on the average vision of both eyes. The different proportion of mediation effect generated by average BCVA and baseline diabetic retinopathy helped to illustrate that were equally important and needed to be carefully taken into consideration.

Another interesting finding regarding visual loss was the patients' performance in color vision. As mentioned previously, the decline in color vision, which was embodied by the ability to choose clothes in NEI VFQ-25, was not associated with the development of T2DM. Moreover, little evidence of either methods such as machinery tests or patients' subjective feedback were included in some studies. 
detected using certain techniques. Additionally, the subtle loss of color vision could be covered by physiological decline in color vision caused by aging, therefore called for early evaluation of color vision and visual complications accompanying T2DM.

Above all, the confirmation of effect driven by different modifiers and the pattern of functional loss in T2DM patients collaboratively promoted the urgent need of early diagnosis, in-time intervention, strict administration and systematic follow-up of all T2DM patients. T2DM patients with multiple risk factors should be paid with more attention and patient-centered therapeutic strategies should be administered. Efficient screening and timely treatment using portable eye examination units guaranteed decreased rate of visual impairments by $86 \%$ in T2DM patients, as reported by a Finnish cohort carried out in more than 14 thousand patients, emphasizing valuable prospective value of taking early action by healthcare professionals ${ }^{[26]}$. Moreover, the absence of intensive blood glucose administration may exacerbate the progression of T2DM. Hu et al reported thatpositive correlation between annual increase of FPG and narrowing of retinal arterioles ${ }^{[27]}$.

There are several limitations to our study. First, it is a cross-sectional study via an online questionnaire, through which a strict causal relationship or quantified correlations between each modifier and functional loss cannot be concluded. Second, quality control was based on the time that each participant spent completing the questionnaire only, which might not be sufficient to prevent certain recall bias. Third, we didn't specifically match every T2DM patient with a non-T2DM participants whose basic information were similar, instead, rough classification of all participants was done according to a 10 -year age range. Therefore, longitudinal data from larger samples was needed to verify 
the above conclusions, and further research might investigate the mechanism and progression of certain ophthalomogic complications and find out certain parameters with predictive values.

To sum up, our study suggested that attention should be paid to the visual function of patients with T2DM, specifically in patients with old age, low educational level, long course of disease, irregular self-monitoring of blood glucose, limited eyesight, and those who had already been diagnosed with diabetic retinopathy. Certain cut-off points were identified, whereas ophthalomogic parameters served as essential mediators of the overall long-term hyperglycemic effect. We emphasized the importance of early diagnosis, in-time intervention and careful administration of T2DM and use more comprehensive parameters to evaluate the severity of visual functiong in T2DM patients.

\section{References}

The Lancet(2020) Diabetes Endocrinology Under the lens: diabetic retinopathy. Lancet Diabetes Endocrinol. 8(11):869. doi: 10.1016/S2213-8587(20)30347-8. Epub 2020 Oct 7. PMID: 33038294.

Ogurtsova K, da Rocha Fernandes JD, Huang Y(2017) IDF Diabetes Atlas: Global estimates for the prevalence of diabetes for 2015 and 2040. Diabetes Res Clin Pract. 128:40-50. doi:10.1016/j.diabres.2017.03.024

Heng LZ, Comyn O, Peto T(2013) Diabetic retinopathy: pathogenesis, clinical grading, management and future developments. Diabet Med. 30(6):640-650. doi:10.1111/dme.12089

Romero-Aroca P, Baget-Bernaldiz M, Pareja-Rios A, Lopez-Galvez M, Navarro-Gil R, Verges R(2016)

Diabetic Macular Edema Pathophysiology: Vasogenic versus Inflammatory. J Diabetes Res.

2016;2016:2156273. doi:10.1155/2016/2156273 
Morrish NJ, Wang SL, Stevens LK, Fuller JH, Keen H(2001) Mortality and causes of death in the WHO Multinational Study of Vascular Disease in Diabetes. Diabetologia. 2001;44 Suppl 2:S14-S21. doi:10.1007/p100002934

Cusick, M., SanGiovanni, J. P., Chew, E. Y., Csaky, K. G., Hall-Shimel, K., Reed, G. F., Caruso, R. C., $\&$ Ferris, F. L., 3rd (2005) Central visual function and the NEI-VFQ-25 near and distance activities subscale scores in people with type 1 and 2 diabetes. American journal of ophthalmology, 139(6), 1042 - 1050. https://doi.org/10.1016/j.ajo.2005.01.008

Mangione, C. M., Lee, P. P., Gutierrez, P. R., Spritzer, K., Berry, S., Hays, R. D., \& National Eye Institute Visual Function Questionnaire Field Test Investigators (2001) Development of the 25-item National Eye Institute Visual Function Questionnaire. Archives of ophthalmology (Chicago, Ill. : 1960), 119(7), 1050 - 1058. https://doi.org/10.1001/archopht.119.7.1050

Hariprasad, S. M., Mieler, W. F., Grassi, M., Green, J. L., Jager, R. D., \& Miller, L. (2008) Vision-related quality of life in patients with diabetic macular oedema. The British journal of ophthalmology, 92(1), 89 - 92. https://doi.org/10.1136/bjo.2007.122416

Trento, M., Passera, P., Trevisan, M., Schellino, F., Sitia, E., Albani, S., Montanaro, M., Bandello, F., Scoccianti, L., Charrier, L., Cavallo, F., \& Porta, M. (2013) Quality of life, impaired vision and social role in people with diabetes: a multicenter observational study. Acta diabetologica, 50(6), $873-877$. https://doi.org/10.1007/s00592-013-0470-1

Araki, A., \& Ito, H. (2009) Diabetes mellitus and geriatric syndromes. Geriatrics \& gerontology international, 9(2), 105 - 114. https://doi.org/10.1111/j.1447-0594.2008.00495.x Song, P., Yu, J., Chan, K. Y., Theodoratou, E., \& Rudan, I. (2018) Prevalence, risk factors and burden of diabetic retinopathy in China: a systematic review and meta-analysis. Journal of global health, 8(1), 
010803. https://doi.org/10.7189/jogh.08.010803

Ben Mehidi, A., Massin, P., Guyot-Argenton, C., Erginay, A., Guillausseau, P. J., \& Gaudric, A. (2003)

La rétinopathie diabétique du sujet jeune: l'enfant et l'adolescent [Diabetic retinopathy in children and adolescents]. Diabetes \& metabolism, 29(3), 300 - 306.

Danet-Lamasou, M., Pérès, K., Matharan, F., Berr, C., Carrière, I., Tzourio, C., Delcourt, C., \& Bourdel-Marchasson, I. (2018) Near Visual Impairment Incidence in Relation to Diabetes in Older People: The Three-Cities Study. Journal of the American Geriatrics Society, 66(4), 699 - 705. de Fine Olivarius, N., Siersma, V., Almind, G. J., \& Nielsen, N. V. (2011) Prevalence and progression of visual impairment in patients newly diagnosed with clinical type 2 diabetes: a 6-year follow up study. BMC public health, 11, 80. https://doi.org/10.1186/1471-2458-11-80

Kajiwara, A., Miyagawa, H., Saruwatari, J., Kita, A., Sakata, M., Kawata, Y., Oniki, K., Yoshida, A., Jinnouchi, H., \& Nakagawa, K. (2014) Gender differences in the incidence and progression of diabetic retinopathy among Japanese patients with type 2 diabetes mellitus: a clinic-based retrospective longitudinal study. Diabetes research and clinical practice, 103(3), e7 - e10. https://doi.org/10.1016/j.diabres.2013.12.043

Ozawa, G. Y., Bearse, M. A., Jr, \& Adams, A. J. (2015) Male-female differences in diabetic retinopathy?. Current eye research, 40(2), 234 - 246. https://doi.org/10.3109/02713683.2014.958500 Maric-Bilkan C. (2017) Sex differences in micro- and macro-vascular complications of diabetes mellitus. Clinical science (London, England : 1979), 131(9), 833 - 846. https://doi.org/10.1042/CS20160998 Foo, V., Quah, J., Cheung, G., Tan, N. C., Ma Zar, K. L., Chan, C. M., Lamoureux, E., Tien Yin, W., Tan, G., \& Sabanayagam, C. (2017) HbA1c, systolic blood pressure variability and diabetic retinopathy 
in Asian type 2 diabetics. Journal of diabetes, 9(2), 200 - 207. https://doi.org/10.1111/1753-0407.12403

Kahloun, R., Jelliti, B., Zaouali, S., Attia, S., Ben Yahia, S., Resnikoff, S., \& Khairallah, M. (2014)

Prevalence and causes of visual impairment in diabetic patients in Tunisia, North Africa. Eye (London,

England), 28(8), 986 - 991. https://doi.org/10.1038/eye.2014.131

Liu, Y., Duan, C., Fang, D., Liu, Y., Xu, H., Zheng, Y., Xuan, Y., Wang, L., Ye, L., Su, R., \& An, M.

(2019) Protective factors for diabetic retinopathy in Type 2 diabetes mellitus patients: Long duration of no less than 10 years. Journal of diabetes and its complications, 33(10), 107383.

https://doi.org/10.1016/j.jdiacomp.2019.05.014

Škrha, J., Šoupal, J., Škrha, J., Jr, \& Prázný, M. (2016) Glucose variability, HbA1c and microvascular complications. Reviews in endocrine \& metabolic disorders, 17(1), $103-110$.

https://doi.org/10.1007/s11154-016-9347-2

Hsieh, Y. T., \& Hsieh, M. C. (2020) Fasting plasma glucose variability is an independent risk factor for diabetic retinopathy and diabetic macular oedema in type 2 diabetes: An 8 -year prospective cohort study. Clinical \& experimental ophthalmology, 48(4), 470 - 476. https://doi.org/10.1111/ceo.13728

Lim, H. B., Shin, Y. I., Lee, M. W., Lee, J. U., Lee, W. H., \& Kim, J. Y. (2020) Association of Myopia with Peripapillary Retinal Nerve Fiber Layer Thickness in Diabetic Patients Without Diabetic Retinopathy. Investigative ophthalmology \& visual science, 61(10), 30.

https://doi.org/10.1167/iovs.61.10.30

Feitosa-Santana, C., Paramei, G. V., Nishi, M., Gualtieri, M., Costa, M. F., \& Ventura, D. F. (2010)

Color vision impairment in type 2 diabetes assessed by the D-15d test and the Cambridge Colour Test. Ophthalmic \& physiological optics : the journal of the British College of Ophthalmic Opticians (Optometrists), 30(5), 717 - 723. https://doi.org/10.1111/j.1475-1313.2010.00776.x 

in ophthalmology, 130(2), 131 - 139. https://doi.org/10.1007/s10633-014-9476-4

461 screening and timely treatment. Acta ophthalmologica, 92(6), $582-587$.

462 https://doi.org/10.1111/aos.12278

463 Hu, Y., Niu, Y., Wang, D., Wang, Y., Holden, B. A., \& He, M. (2015) The association of longitudinal 464 trend of fasting plasma glucose with retinal microvasculature in people without established 465 diabetes. Investigative ophthalmology \& visual science, 56(2), $842-848$. 


\section{Supplementary Files}

This is a list of supplementary files associated with this preprint. Click to download.

- Supplementaryfiles.pdf 